\title{
Distributed Control of PEV Charging Based on Energy Demand Forecast
}

\author{
Mithat C. Kisacikoglu ${ }^{(0)}$, Member, IEEE, Fatih Erden, Member, IEEE, and Nuh Erdogan, Member, IEEE
}

\begin{abstract}
This paper presents a new distributed smart charging strategy for grid integration of plug-in electric vehicles (PEVs). The main goal is to smooth the daily grid load profile while ensuring that each PEV has a desired state of charge level at the time of departure. Communication and computational overhead, and PEV user privacy are also considered during the development of the proposed strategy. It consists of two stages: 1) an offline process to estimate a reference operating power level based on the forecasted mobility energy demand and base loading profile, and 2) a real-time process to determine the charging power for each PEV so that the aggregated load tracks the reference loading level. Tests are carried out both on primary and secondary distribution networks for different heuristic charging scenarios and PEV penetration levels. Results are compared to that of the optimal solution and other state-of-the-art techniques in terms of variance and peak values, and shown to be competitive. Finally, a real vehicle test implementation is done using a commercial-ofthe-shelf charging station and an electric vehicle.
\end{abstract}

Index Terms-Distributed control, grid integration, peak shaving, plug-in electric vehicle (PEV), smart charging.

\section{INTRODUCTION}

G ROWING number of plug-in electric vehicles (PEVs) in the market is becoming a matter of concern due to the utility grid integration, especially at the distribution level [1] [3]. The integration of PEVs into the distribution system with an uncoordinated fashion at high market rates increases peak loading on line/transformer, energy losses, voltage deviations, and the need for network reinforcements [1]-[5]. When considering a cost-efficient solution for both the utility grid and PEV user, it is more convenient to shift PEV charging loads to off-peak hours where the demand load and the electricity price are lower. However, shifting PEV loads with uncoordinated charging

Manuscript received December 14, 2016; revised April 17, 2017; accepted May 1, 2017. Date of publication May 17, 2017; date of current version January 3, 2018. Paper no. TII-16-1524. (Corresponding author: Mithat Kisacikoglu.)

M. C. Kisacikoglu is with the Department of Electrical and Computer Engineering, University of Alabama, Tuscaloosa, AL 35487 USA (e-mail: mkisacik@ua.edu).

F. Erden is with the Department of Electrical and Electronics Engineering, Bilkent University, Ankara 06800, Turkey (e-mail: erden@ ee.bilkent.edu.tr).

N. Erdogan is with the Department of Electrical Engineering, University of Texas at Arlington, Arlington, TX 76019 USA (e-mail: nuh. erdogan@uta.edu).

Color versions of one or more of the figures in this paper are available online at http://ieeexplore.ieee.org.

Digital Object Identifier 10.1109/TII.2017.2705075 strategies imposes a nonuniform load profile resulting in undesired peak loads at off-peak hours due to the charging many PEVs simultaneously [6].

The peak loads caused by PEV charging can be restrained either by unidirectional PEV charging management [4], [6] or by discharging PEV batteries into the grid using vehicle-togrid (V2G) technology [7], [8]. V2G requires special hardware allowing bidirectional power transfer, which is currently not available in the most of the market vehicles yet. Also, the lack of an established electric vehicle grid integration (EVGI) protocol and market agreement make it difficult to provide mass field deployment for V2G technology. On the other hand, unidirectional charging coordination can be realized with low-level control utilizing IEC 61851/SAE J1772 standards which are already applicable at market vehicles [9], [10]. Therefore, coordinated unidirectional charging becomes prominent for large-scale penetration of PEVs into the grid in the near term.

Coordinated charging manages PEV charging loads effectively to mitigate largely undesirable impacts of high penetration of PEVs into the grid [4], [5]. It enables a charging flexibility which can be used to provide grid services such as peak shaving [6], valley filling [11], and minimizing charging costs [12]. It can also be used to integrate higher share of intermittent renewable energy sources into the grid [13]. Centralized [13], [14] and distributed (decentralized) [11], [12], [15]-[18] charging strategies have been proposed in the literature. While charging profile for each PEV in the centralized strategy is managed by a central operator which aims to achieve an optimal aggregated charging goal, the distributed strategy allows each PEV to determine its own charging profile which may not always result in optimal aggregated charging regime [12]. However, distributed approach has gained more attraction in the literature because of its higher flexibility to the PEV user, higher reliability, and easier field implementation [11], [12], [15]-[18]. On the other hand, better utility-grid coordination while considering PEV user convenience is still an undergoing research topic.

Most of the coordinated charging strategies assume rated charger power for charging the PEV batteries [11], [15]-[17], [19]. The strategy followed in these cases is to schedule the PEVs by considering the load profile and the energy demand of each PEV. However, the flexibility in scheduling the PEVs considerably decreases at low penetration levels which in turn results in a notable decrease in valley filling/peak shaving performance. On the other hand, coordinated charging strategies that assume variable rates can contribute more by considering the load profile and adjusting the charging rates accordingly for 
each PEV in real time. Furthermore, variable charging at integer current rates can be seamlessly implemented with the current EVGI technology. PEVs are capable of responding to any remote charging step change command in less than $10 \mathrm{~s}$ making them suitable for variable charging control with sample time as low as $1 \mathrm{~min}[20]$.

Charging strategies with variable power rates can be exploited better for demand side management [21] when compared to charging at rated power. As such, the flexibility in charging power helps us to better match PEV charging loads with intermittent renewable generation [13], [22], as well as dynamic electricity prices for optimizing the charging cost [23]. The studies addressing the charging strategies with variable power rates [12], [24], [25], assume a constant charging trajectory (reference line), which is tracked by the individual PEV charging algorithms. The main drawback of these studies is that the grid load profile and PEV mobility are assumed to be fundamentally deterministic. This is clearly not the case in practice, as the demand load and PEVs' mobility are stochastic. Also, the algorithms have extensive computations and broadcast messages that require bidirectional communication in each iteration between the grid and PEVs. This is a main barrier for their application in realtime environment. Moreover, the PEV penetration rates have definitely an impact on the valley-filling performance which has not been investigated in [12] and [24]. Therefore, coordinated charging strategies which can perform a flat aggregated load profile at different loading with various PEV mobility characteristics and penetration rates should be further researched.

This study contributes to the integration of PEVs into the distribution system by proposing a new distributed smart charging algorithm. The proposed algorithm reduces the peak loading on the distribution system, fills the night-valley as much as possible while ensuring the desired state of charge (SOC) level at departure time. In contrast to the charging at constant rated power proposed in [11], [15]-[17], charging process for each PEV is performed at variable power rates to achieve better valley filling performance. For this purpose, a new two-stage control approach is introduced. First, an offline process is followed to determine the charge reference based on the model of PEV users' travel behaviors and base load profile forecast. Second, a real-time operation is carried out with the distributed control approach. The algorithm not only provides a near-optimal aggregated load profile to minimize the variance of overall loading, but also utilizes the advantages of distributed strategy, i.e., retains PEV user private information and avoids the communication and computation overhead. The designed charging strategy requires only one message broadcasting between the grid and PEV at the time a PEV is connected to the grid. Furthermore, the calculation of charging power for each PEV is based on simple arithmetic operations without need to solve any optimization problem, which makes it suitable to be realized with an embedded system within the PEV. The proposed charging strategy is tested on real distribution, and upstream networks for different heuristic charging scenarios and PEV penetration rates. The performance of the algorithm is quantified in terms of variance and mean square error (MSE) metrics. Finally, the developed algorithm is implemented on a real electric vehicle utilizing IEC 61851 compatible charging station and charging plug.

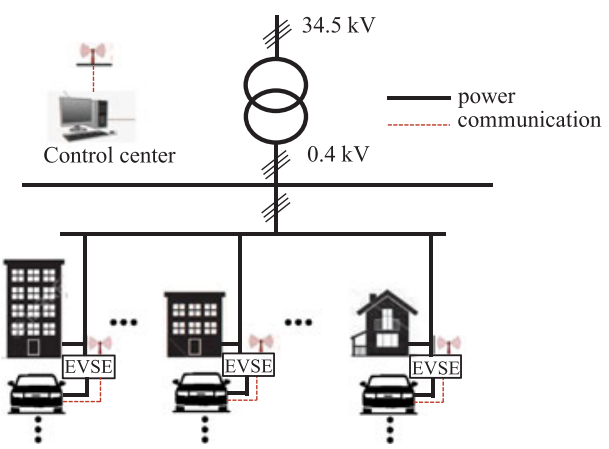

Fig. 1. EVGI architecture in the distribution system.

TABLE I

TYPES OF PEVS AND THEIR SPECIFICATIONS

\begin{tabular}{lcccc}
\hline \hline $\begin{array}{l}\text { Vehicle Make } \\
\text { and Model }\end{array}$ & $\begin{array}{c}\text { Vehicle } \\
\text { Type }\end{array}$ & $\begin{array}{c}\text { Usable Batt. } \\
\text { Size (kWh) }\end{array}$ & $\begin{array}{c}\text { EV Range } \\
(\mathrm{mi})\end{array}$ & $\begin{array}{c}\text { Max. Onboard } \\
\text { Charging Power (kW) }\end{array}$ \\
\hline BMW i3 & EV & 18.8 & 81 & 7.4 \\
Chevrolet Volt & PHEV & 14 & 53 & 3.3 \\
Nissan Leaf & EV & 30 & 107 & 6.6 \\
Chevrolet Bolt & EV & 60 & 238 & 7.2 \\
Tesla Model S & EV & 70 & 240 & 10 \\
\hline \hline
\end{tabular}

The rest of this paper is organized as follows. Section II introduces the system framework and modeling. Section III describes the optimal solution and the development of the distributed control algorithm. Case studies and experimental test implementation are presented in Sections IV and V, respectively. Finally, the main conclusion remarks are given in Section VI.

\section{SYSTEM DESCRIPTION AND MOdELING}

In this study, system modeling is done in MATLAB through offline and time-based simulation environments. System architecture is demonstrated in Fig. 1. The distribution grid is a three-phase, $400 \mathrm{~V}$ 1-1, 1000-kVA test system where the individual residential loads are supplied with single-phase $230 \mathrm{~V}$ utility power source. The components of the system model are individually described in the below sections.

\section{A. Transportation Mobility Modeling}

To determine a reference loading level, which will be explained in Section III, the PEV users' travel behaviors should be modeled. The more we know about the home arrival/departure times and energy needs of PEVs, the more accurate the selected reference level will be. To the best of our knowledge, there is no available data on the driving patterns of vehicle owners in Turkey provided by Turkish Statistical Institute. Thus, the daily trip data of ten personal vehicle owners are collected for about a year to build a realistic mobility model for this study. These data belong to daily personal usage of management staff at Başkent DisCo, and it is collected using vehicle tracking devices [26]. Histograms of the collected data turns out to be quite similar to a Gaussian distribution with mean and standard deviations of (7h47, 0h23), (19h55, 1h40), and (39.5 km, $15.8 \mathrm{~km})$ for home departure time, arrival time, and daily trip distance distributions, respectively. Five different PEVs listed in Table I are 
randomly selected in the model. The transportation model runs offline and generates SOC values for each PEV at the time of grid connection based on the model parameters.

\section{B. Charging Station Model}

Charging station (electric vehicle supply equipment-EVSE) model includes a user behavior model which is defined as follows:

1) Standard charging: PEV charges at rated on-board charging power when connected to the grid. No control over charging is possible. Charging ends when the PEV is fully charged.

2) Smart charging: PEV starts charging with a smart charging profile during the time when the system loading is below the reference value. Charging ends in the morning departure time with fully charged battery.

The EVSE communicates with the control center using a wireless/wired setup. Vehicle-station communication is employed through the low-level communication over the control pilot $(\mathrm{CP})$. The amount of current thereby active power for battery charging is determined by the duty cycle ratio $D$ of the $\mathrm{CP}$ which is a $1-\mathrm{kHz}$ square-wave signal oscillating between $+6 \mathrm{~V}$ and $-12 \mathrm{~V}$ during charging as described in IEC 61851/ SAE J1772 [9], [10]. This signal pin is available at every charging plug and vehicle inlet. As defined in the standards, a unidirectional charging control scenario can be applied by adjusting $D$ as follows:

$$
I= \begin{cases}0 & \text { for } \quad D<10 \% \\ D \times 0.6 & \text { for } \quad 10 \% \leq D \leq 83.3 \% \\ (D-64) \times 2.5 & \text { for } \quad D>83.3 \%\end{cases}
$$

where $I$ is the ac rms charger current (A). Based on (1), the minimum positive charging current $I$ is 6 A, i.e., $D=10 \%$, and $I=10 \times 0.6=6 \mathrm{~A}$.

\section{On-Board Charger and Battery System Model}

The PEV component models are developed to simulate the ac power exchange between the vehicle and the grid. It includes the following information for different vehicle vendors: on-board charger power rating (listed in Table I), operating efficiency, implementation of constant-current (CC), and constant-voltage (CV) charging, and cell-based battery system design. On-board chargers are assumed to operate at a 90\% efficiency with 1.0 power factor.

A 24.8 Ah, 3.7 V nominal, baseline Li-ion battery cell is used to model the PEV batteries listed in Table I using various cell configurations to meet the nameplate battery energy capacity. Fig. 2 shows the model of the battery cell used in this study. This cell model can capture the impact of battery SOC calculation on the performance of the developed charging algorithm which is in the scale of minutes. Battery SOC is computed by measuring the current, thereby charge, entering and leaving the battery as follows:

$$
\mathrm{SOC}=\frac{Q_{0} \pm \int i_{b t} d t}{Q_{n}} \times 100
$$

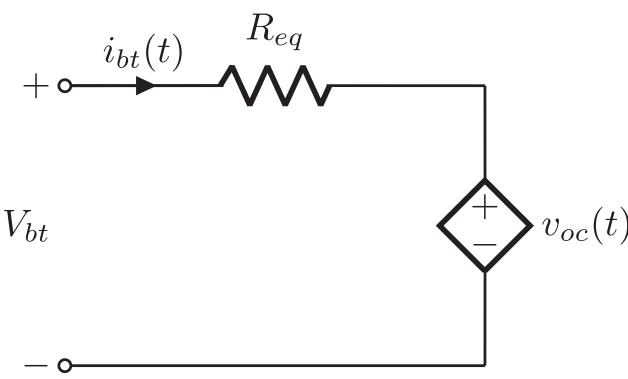

Fig. 2. Equivalent model of a battery cell.



Fig. 3. Li-ion cell characteristics used for modeling battery systems: cell voltage versus SOC (circle, blue) and equivalent resistance versus SOC (square, red)

where $Q_{o}$ is the initial electric charge present before charging/discharging the battery (C), $Q_{n}$ is the nominal electric charge capacity of the battery $(\mathrm{C})$, and $i_{b t}$ is the battery charging current (A). $i_{b t}$ can be either negative or positive depending on the current direction, i.e., in this study it is only positive. Note that SOC computed with (2), also known as coulomb counting, is used as a measure of battery energy in a scale of $0-100 \%$.

The open-circuit voltage and the equivalent series resistance $R_{\text {eq }}$ both depend on the computed SOC values as described in Fig. 3. The terminal voltage $V_{b t}$ is important to determine when to switch from $\mathrm{CC}$ to $\mathrm{CV}$ charging. Here, when $V_{b t}$ reaches $4.0 \mathrm{~V}$, the charge cycle switches to $\mathrm{CV}$ charging. The battery cells are assumed to be equally charged with proper equalization by the battery management system.

\section{Distributed Smart Charging Algorithm}

\section{A. Optimal Charging Control}

We start by finding the optimal charging profiles of PEVs to provide a basis for assessing the performance of the developed algorithm. Optimality criterion is a flat aggregated (base and PEV charging) load profile. However, it is not always achievable because the grid load and PEV mobility data are stochastic in nature, and this limits the flexibility in PEV charging control. Considering a forecasted based load and PEV mobility data, a preferred operating point (POP) can be determined. Then, the objective becomes to track this POP value. Thus, PEV charging procedure can be formulated as an optimal charging control problem whose objective is to minimize the MSE between the aggregated load profile and the predefined POP value. 
Let us consider a $24 \mathrm{~h}$ time horizon divided into a $T$ number of time slots of $1 \mathrm{~min}$ each. Let $P_{c h, i}=\left\{P_{c h, i}(1) \ldots P_{c h, i}(T)\right\}$ denote the charging profile of the $i^{\text {th }} \mathrm{PEV}$, and $n$ be the number of PEVs. $P_{\text {base }}(t)$ and $P_{c h, i}(t)$ denote the grid base load and charging profile of the $i^{\text {th }} \mathrm{PEV}$ at time $t$, respectively. $t_{\mathrm{arr}, i}$ and $t_{\mathrm{dept}, i}$ are the arrival and departure times of the $i^{\text {th }} \mathrm{PEV}$, and $t_{s, i}$ and $t_{e, i}$ are the beginning and end of the charging period for the $i^{\text {th }} \mathrm{PEV}$, respectively. The objective function can be expressed as follows:

$$
\begin{aligned}
\underset{P_{c h, 1} \ldots P_{c h, n}}{\min } & \sum_{t=1}^{T}\left(P_{\text {base }}(t)+\sum_{i=1}^{n} P_{c h, i}(t)-\mathrm{POP}\right)^{2} \\
\text { subject to } & P_{\min } \leq P_{c h, i}(t) \leq P_{c h, i}^{\mathrm{rated}} \forall t \in\left[t_{s, i}, t_{e, i}\right] \\
& P_{c h, i}(t)=0 \forall t \notin\left[t_{s, i}, t_{e, i}\right] \\
& t_{\mathrm{arr}, i} \leq t_{s, i}<t_{\mathrm{dept}, i} \\
& t_{\mathrm{arr}, i}<t_{e, i} \leq t_{\mathrm{dept}, i} \\
& \sum_{e, i} P_{c h, i}(t) \times \Delta t / 60 \\
& \sum_{t=t_{s, i}} \\
& =\left(1-\mathrm{SOC}_{\mathrm{arr}, i}\right) \times E_{\mathrm{rated}, i} .
\end{aligned}
$$

Equation (3) always seeks optimal charging rates as PEVs connect to the grid, while the constraints must be satisfied simultaneously. Minimizing the MSE aims to have an aggregated load profile tracking the POP, which in turn, results in a valley-filling behavior. The first constraint states that the charging power is between a minimum and maximum rated value in compliance with the standards IEC 61851/ SAE J1772 [9], [10]. The second constraint ensures the charging operation takes places within the start and end charging times. The last constraint ensures every PEV has $100 \%$ SOC at departure time.

The optimization problem is solved in a decentralized fashion. That is, the optimal charging profile of each PEV is locally found by the custom solution of (3) at the time the PEV is first pluggedin. Then, the PEV sends its charging profile back to the grid, and the operator updates the grid load profile. The PEVs do not necessarily update their charging profiles as new PEVs connect to the grid. However, the computational intensity of the iterative algorithm in finding the optimal solution makes it impractical for real-time implementation. Also, the optimal solution requires the design of EVSEs with fast signal processing units which would increase the upfront cost.

In this study, we propose a distributed control strategy which significantly reduces the computational load and makes the management of the charging control more practical for field implementation. The proposed strategy consists of two stages, namely, offline and real-time processing, as described in Fig. 4. During the offline process, a POP value is determined based on the forecasted base load and mobility data. In the second phase, a real-time operation is carried out when each PEV is connected to the grid as follows:

1) The grid operator sends the valley power profile based on forecasted load and actual mobility data to $i^{\text {th }} \mathrm{PEV}$ connected to the grid;

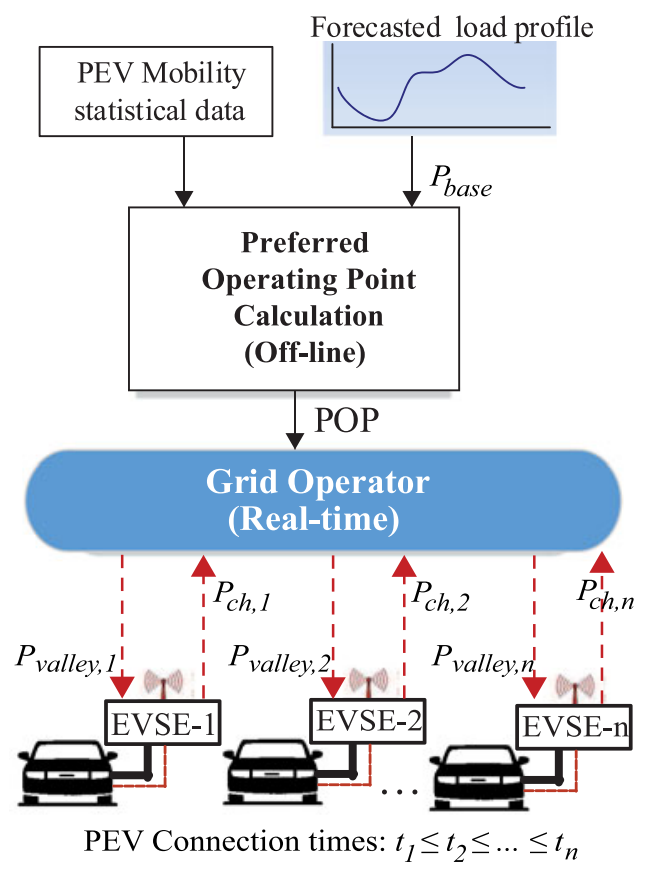

Fig. 4. Proposed charging control framework.

2) the PEV independently determines its own charging profile depending on the valley energy, its SOC, and morning departure time, and then sends it to the grid operator;

3 ) the grid operator updates valley power and sends it to $(i+1)$ th PEV connected to the grid.

The algorithm repeats the second and third steps whenever a new PEV is connected to the grid.

\section{B. Proposed Distributed Charging Control}

1) Offline Operation: This stage aims to find a good POP value for the grid when the PEVs are included in the scenario. In the online process, the POP value will be used to define the valley energy, and charging power of each PEV will be determined such that the PEVs all together fill the valley. The total number of PEVs in the region, their types (makes/models), and their mobility parameters are assumed to be well estimated within an error percentage. Also, it is assumed that the load profile is forecasted with a reasonable accuracy (within an error) based on statistical analysis of historical data [27]-[29]. The prediction methods employed for these forecasting events are not addressed in this study.

First, a mobility dataset (daily distances taken, home arrival, and departure times) is generated based on the predetermined Gaussian characteristics defined in Section II. Then, by considering the battery capacities of each PEV and the distances taken, required energy for an SOC level of $100 \%$ is computed. As a final step, total required energy is compared to the current valley energy and the POP value is updated accordingly until a convergence criterion is satisfied. The offline process is summarized in Algorithm 1. 




The valley energy in Algorithm 1 is defined as follows:

$$
E_{\text {valley }}=\int_{t_{\text {arr }, 1}}^{t_{\text {dept } \mathrm{ave}}} P_{\text {valley }}(t) d t
$$

where

$$
P_{\text {valley }}(t)= \begin{cases}\mathrm{POP}-P_{\text {base }}(t), & \text { if POP }-P_{\text {base }}(t)>0 \\ 0, & \text { otherwise }\end{cases}
$$

and $t_{\mathrm{arr}, 1}$ is the time the first PEV arrives home, and $t_{\mathrm{dept} \text {,ave }}$ is the average of the departure times of all PEVs. Among possible different boundary selections for the limits of integral in (4), $t_{\mathrm{arr}, 1}$ and $t_{\mathrm{dept,ave}}$ are selected as they gave the best performance in minimizing the variance of the aggregated load. To have a good representation of a global POP value, the above procedure is repeated 100 times, and the mean of the results is fed to the online process as the final POP value.

2) Online Operation: Once a good estimate for the POP value is determined, the only information to be transferred to PEVs to determine the charging profile for each PEV is the $P_{\text {valley }}(t)$ which is calculated using (5). To determine the charging power of a PEV at time $t$, the valley energy for that PEV is defined first

$$
E_{\text {valley }, i}(t)=\int_{t}^{t_{\text {dept }, i}} P_{\text {valley }}(\tau) d \tau \quad \forall t \in\left[t_{\text {arr }, i}, t_{\text {dept }, i}\right] .
$$

Note that the limits of the integral in (6) are different than the ones in (4) and set to the exact arrival and departure times of the $i^{\text {th }}$ PEV. Then, a multiplication factor $\alpha(t)$ is computed as

$$
\alpha_{i}(t)= \begin{cases}\frac{E_{\mathrm{rated}, i} \times\left\{1-\mathrm{SOC}_{i}(t)\right\}}{E_{\text {valley }, i}(t)} & \forall t \in\left[t_{\mathrm{arr}, i}, t_{\mathrm{dept}, i}\right] \\ 0, & \text { otherwise }\end{cases}
$$

where $E_{\mathrm{rated}, i}$ is the rated battery capacity of the $i^{\text {th }}$ PEV. Finally, the charging power for the $i^{\text {th }} \mathrm{PEV}$ at time $t$ is

$$
P_{c h, i}(t)=\alpha_{i}(t) \times P_{\text {valley }}(t) .
$$

In this study, the charging control signals for each PEV are compliant with the IEC 61851/SAE J1772 standards which impose that the charging current has to be at least $6 \mathrm{~A}$ [9], [10]. For this purpose, if $P_{c h, i}$ in the above equation results in a value below the possible minimum charging power $(1.38 \mathrm{~kW}$ at $230 \mathrm{~V})$, then it is updated as indicated in (9). Further, the charging power is also checked against the on-board rated charging power listed in Table I.

$$
P_{c h, i}(t)=\left\{\begin{array}{lll}
0 \mathrm{~kW} & \text { if } \quad 0 \leq P_{c h, i} \leq 0.69 \mathrm{~kW} \\
1.38 \mathrm{~kW} & \text { if } \quad 0.69 \mathrm{~kW}<P_{c h, i} \leq 1.38 \mathrm{~kW} \\
P_{\text {rated }, i} & \text { if } \quad P_{c h, i} \geq P_{\text {rated }, i} .
\end{array}\right.
$$

At the end of time $t, \mathrm{SOC}_{i}(t)$ is updated accordingly and the above procedure is repeated between (7) and (9) to find the charging power for the same vehicle at time $t+1$. Having determined $P_{c h, i}(t)$ for the whole time period when the $i^{\text {th }} \mathrm{PEV}$ is parked, this information is sent back to the grid, and $P_{\text {valley }}(t)$ is updated. That is,

$$
P_{\text {valley }}(t)=P_{\text {valley }}(t)-P_{c h, i}(t) . \quad \forall t \in[0,24 h] .
$$

The charging power for subsequent PEV connected to the grid is determined based on this new value of the valley power. Note that the updated valley power is set to zero for those time instances where it takes negative values. This, in turn, prevents $P_{c h, i}(t)$ from taking negative values.

This control approach has several advantages in terms of computational complexity, communication overhead, and practicability for real-time applications. Since the charging calculations are distributed to EVSEs, and the charging profile for each PEV is determined in a noniterative manner, the communication overhead is decreased. It is calculated only once when the PEV connects to the grid, and does not need to be updated depending on the other PEVs arriving later. From PEV user privacy perspective, the control approach preserves user private data since PEVs report only charging profiles and do not share user-specific vehicle/departure time information. The solutions of the charging profile expressions do not require extensive calculations. The charging expressions can be easily solved by an embedded system in each EVSE which are further described in experimental study in Section V. Therefore, the control approach is quite appropriate for field implementation.

\section{Case Studies and Test Results}

\section{A. Case Studies on a Residential Distribution Network}

Case studies are developed using a part of a large distribution network in a metropolitan city [26]. A three-phase distribution transformer of $34.5 / 0.4 \mathrm{kV}, 1000 \mathrm{kVA}$, with 985 residential customers are used. The daily average active power profile during four months are shown in Fig. 5. It is important to note that the most common heating method in the selected area is burning natural gas (NG) which considerably decreases power consumption. Also, the $1 \mathrm{~h}$ time difference for the start of peak-loading between Sept-Oct and Nov-Dec is due to daylight savings time. Among the available months, November is chosen as the forecasted daily power consumption. The triple tariff regions $(1,2$, and 3) shown in the figure correspond to night (10 P.M.-6 A.M.), day-time (6 A.M.-5 P.M.), and peak-time (5 P.M.-10 P.M.) hours, 


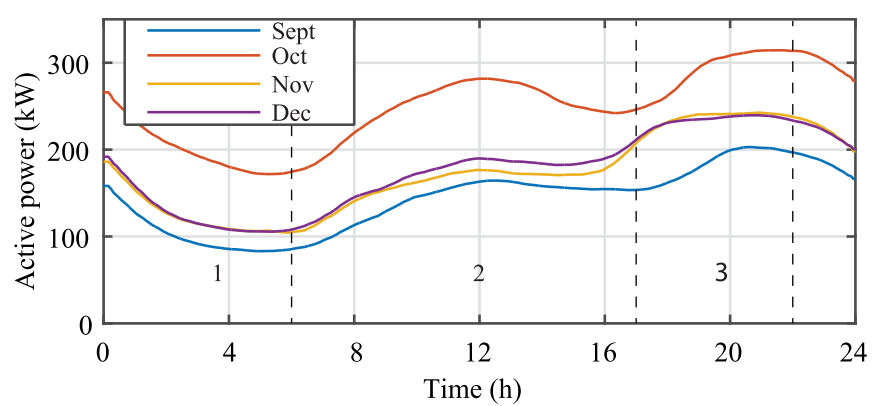

Fig. 5. Daily average active power demands for four months (non-EV).

respectively. The proof of concept is demonstrated for the PEV penetration level of $10 \%$. This corresponds roughly to $100 \mathrm{ve}-$ hicles for 985 customers in the neighborhood assuming each household owns one vehicle.

In order to evaluate and compare the operation results, variance is used as the base metric. Minimizing the variance shows how well the algorithm smooths the aggregated load profile helping for the better utilization of the power generation assets. The variance is calculated as follows:

$$
v=\frac{1}{t_{\text {dept,ave }}-t_{\text {arr,ave }}} \sum_{t=t_{\text {arr ave }}}^{t_{\text {dept ave }}}\left\{P_{\text {aggr }}(t)-\mu\right\}^{2}
$$

where

$$
P_{\text {aggr }}(t)=P_{\text {base }}(t)+\sum_{i=1}^{n} P_{\mathrm{ch}, i}(t)
$$

and

$$
\mu=\frac{1}{t_{\mathrm{dept}, \mathrm{ave}}-t_{\mathrm{arr}, \mathrm{ave}}} \sum_{t=t_{\mathrm{arr}, \mathrm{ave}}}^{t_{\mathrm{dept}, \mathrm{ave}}}\left\{P_{\mathrm{base}}(t)+\sum_{i=1}^{n} P_{\mathrm{ch}, i}(t)\right\}
$$

where $P_{\mathrm{ch}, i}(t)$ is the charging load $(\mathrm{kW})$ of the $i^{\text {th }}$ PEV at time $t$. The calculations are done in 1-min intervals during the time horizon when vehicles are parked and grid connected at home. The average arrival and departure times of the vehicle set are $t_{\text {arr,ave }}=7: 55$ P.M. and $t_{\text {dept,ave }}=7: 47$ A.M. As a second measure, MSE can also be used. It is a measure of how close the aggregated loading profile is to the expected charge reference (POP value). This metric can be used when a PEV aggregator promises to provide a load consumption service to the utility grid in a predefined time interval, i.e., $t_{1}=1: 00$ A.M. to $t_{2}=7: 47$ A.M. The MSE is computed as follows:

$$
\mathrm{MSE}=\frac{1}{t_{2}-t_{1}} \sum_{t=t_{1}}^{t_{2}}\left(P_{\mathrm{aggr}}(t)-\mathrm{POP}\right)^{2} .
$$

We first compare the smart charging algorithm with the optimal solution. The optimal solution is obtained in MATLAB using the convex optimization toolbox CVX [30]. The simulations are run for 100 times to cover a considerable number of scenarios and to evaluate the performance of the algorithms. The presented figures show the averaged results among 100 simulation runs.

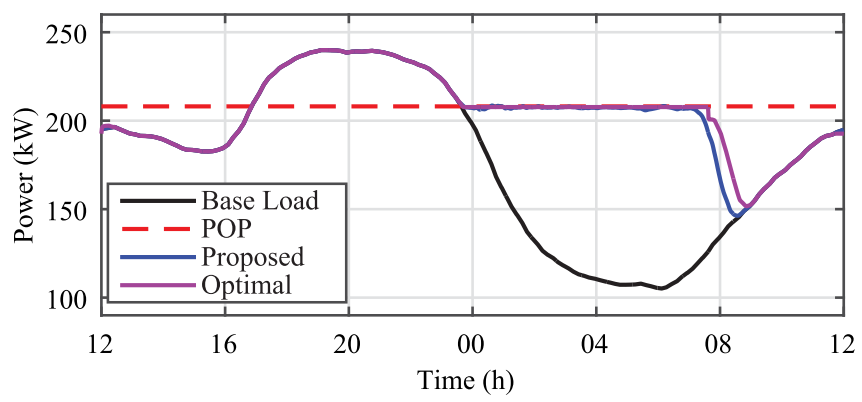

Fig. 6. Aggregated load profile with proposed and optimal charging algorithms for $10 \%$ PEV penetration.

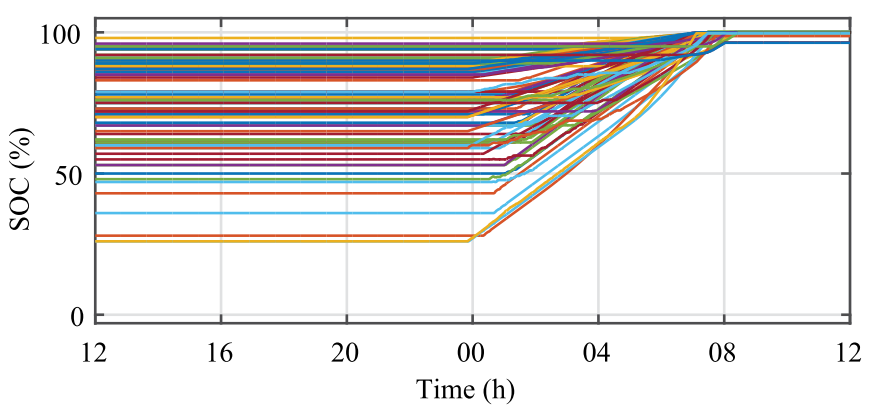

Fig. 7. SOC change during the proposed charging process.

TABLE II

Performance of Charging Algorithms on the DISTRIBUTION NETWORK

\begin{tabular}{lcc}
\hline \hline Algorithm & $\begin{array}{c}\mathrm{MSE}^{*} \\
(\mathrm{~kW})^{2}\end{array}$ & $\begin{array}{c}\text { Variance }^{\dagger} \\
(\mathrm{kW})^{2}\end{array}$ \\
\hline Standard Charging & N/A & 13138 \\
Optimal Charging & 3.68 & 150.81 \\
Proposed Smart Charging & 12.98 & 165.65 \\
\hline \hline
\end{tabular}

${ }^{*}$ Calculated between 1:00 A.M. and 7:47 A.M. ${ }^{\dagger}$ Calculated between 7:55 P.M. and 7:47 A.M. next day.

Fig. 6 presents the behavior of the proposed and optimal algorithms on the distribution network for 10\% PEV penetration. Both algorithms can track the POP during the charging time interval, and can provide a satisfactory valley-filling performance. Charging loads are successfully shifted toward off-peak hours such that peak loading of the transformer is not increased. The change in SOC during charging is shown in Fig. 7 for every PEV. All PEVs end up with more than 95\% SOC at departure time. The proposed algorithm falls short of providing exactly $100 \%$ SOC to all PEVs. However, it is achieved by the optimal solution.

The MSE and variance values obtained by each algorithm are reported in Table II. Compared to standard charging, the optimal and proposed algorithms reduce the variance significantly. The optimal solution returns the best overall utilization of the generation sources without causing high demand charging loads for the utility operator while the standard charging returns the worst utilization of the assets. The proposed algorithm gives 


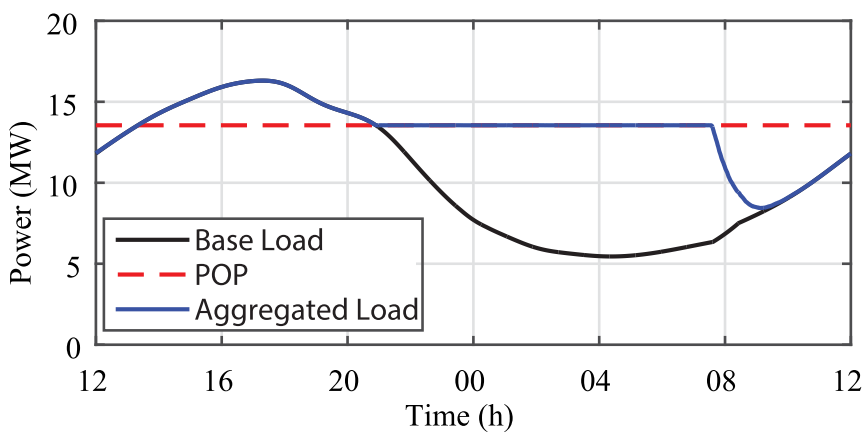

Fig. 8. Summer load profile with 50\% PEV penetration.

a near-optimal solution. It produces an MSE rate close to that of the optimal solution. The optimal solution gives the lowest MSE and variance values, but it is impractical in field implementation due to the computational intensity of its algorithm. Overall, the proposed control algorithm handles EVGI impact on the distribution network successfully and provides a smooth and constant loading profile by determining a corresponding charging reference to be followed by the PEVs.

\section{B. Case Studies on Upstream Network}

This case study is used to assess the performance of the proposed charging algorithm on the upstream network with a high PEV penetration rate (i.e., 50\%). The load profiles and PEV penetration rate in this case study are also selected on purpose to compare the performance of our algorithm with the distributed charging algorithm proposed by Binetti et al. [11]. For this comparison, the daily average residential loading composed of 10000 houses in the Southern California Edison area is considered as the base load profile. The load profiles in summer [31] and winter [32] seasons are used separately since the peak/off-peak demand and timing differ in each case. The average number of PEVs per household is taken as 1.86 based on [33]. Thus, the corresponding number of PEVs for 50\% PEV penetration rate is 9300 [11]. PEV mobility data are assumed to follow a Gaussian characteristic. The mean and standard deviations for plug-in, plug-off times, and SOC level distributions are $(17 \mathrm{~h} 00,2 \mathrm{~h} 00),(07 \mathrm{~h} 00,1 \mathrm{~h} 00)$, and $(50 \%, 10 \%)$, respectively, as reported in [11]. The desired departure SOC is set to $80 \%$ as in [11].

The resulting diagrams for the proposed algorithm on the summer and winter load profiles with 50\% PEV penetration rate are shown in Figs. 8-9, respectively. As illustrated in Fig. 8, the smart charging algorithm achieves a very good valley-filling performance with a low variance value. The algorithm reduces the variance of the base load profile more than three times, and does not increase the peak load. The peak value of the aggregated load profile has decreased almost by $60 \%$ compared to standard charging case given in Table III. Moreover, the charging algorithm achieved a reduced variance value compared to standard charging case in winter load profile case as shown in Fig. 9. In the same load profile, the peak value of the base load profile slightly increased. In fact, this peak value is unavoidable

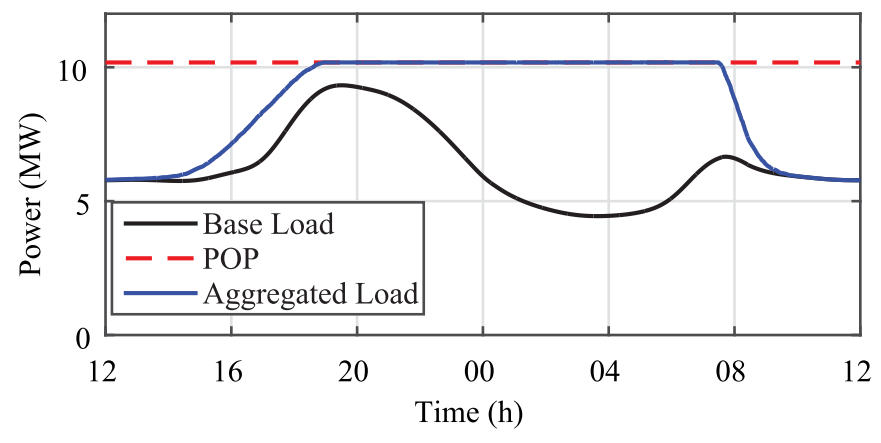

Fig. 9. Winter load profile with 50\% PEV penetration.

TABLE III

COMPARISON OF THE ALGORITHM's PERFormance ON UPSTREAM NETWORK

\begin{tabular}{lccccc}
\hline \hline & \multicolumn{2}{c}{ Aggregated profile } & & \multicolumn{2}{c}{ Aggregated profile } \\
\cline { 2 - 3 } \cline { 5 - 6 } & \multicolumn{2}{c}{ w/ summer base load } & & \multicolumn{2}{c}{ w/ winter base load } \\
\cline { 2 - 3 } \cline { 5 - 6 } & Variance & Peak value & & Variance & Peak value \\
Algorithm & $(\mathrm{MW})^{2}$ & $(\mathrm{MW})$ & & $(\mathrm{MW})^{2}$ & $(\mathrm{MW})$ \\
\hline Base load & $12,856,464$ & 16.3 & & $1,575,052$ & 9.3 \\
Stand. charging & $44,478,243$ & 27.5 & & $23,535,472$ & 21.4 \\
Distrib. algor.* & $5,119,561$ & 16.3 & & $4,911,268$ & 10.7 \\
Propos. algor. & $3,704,735$ & 16.3 & & $3,505,015$ & 10.2 \\
\hline \hline
\end{tabular}

*The data is taken from Table I in [11].

since the valley energy is not sufficient to charge the PEVs fully. However, the achieved peak value is very much less than that of standard charging case. Therefore, the performance of the algorithm can also be assessed as satisfactory on the winter load profile with high penetration case (50\%).

Table III presents a comparison of the proposed algorithm with standard and state-of-the-art distributed charging algorithms presented in [11]. The proposed charging algorithm outperforms the charging algorithm presented in [11] in terms of variance and peak value of the aggregated load profile. The algorithm reduced the variance by approximately $30 \%$ compared to the values reported in [11] for both summer and winter load profiles. Also, the achieved peak value for winter load profile is $5 \%$ lower than the result reported in [11].

\section{Impact of Possible Error Sources}

In this section, we evaluated the performance of the proposed algorithm when there are forecasting errors on the case study discussed in Section IV-A. The following possible errors have been considered as baseline error analysis:

1) Mobility parameters: PEVs arrive/leave home according to Gaussian distributions whose means are shifted by 5 min compared to the models built from the historical data. In addition, the mean of the actual daily distance distribution is assumed to be $5 \mathrm{~km}$ less than the expected.

2) Number of PEVs: There are ten less PEVs than estimated yielding a $10 \%$ error in the total number of EVs. 


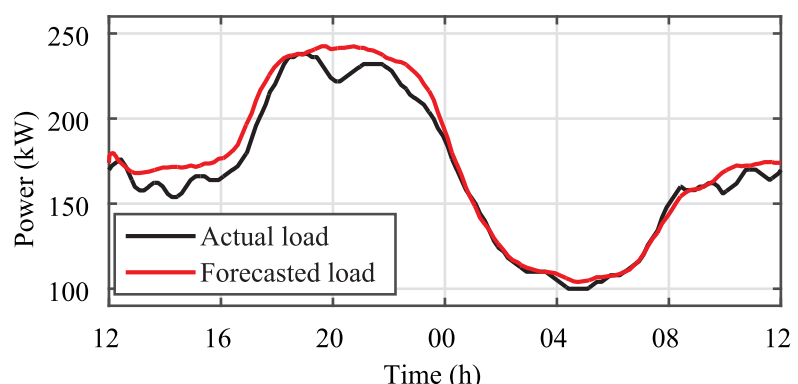

(a)



(b)

Fig. 10. Behavior of the algorithm due to load forecasting error: (a) Forecasted load and actual load, (b) load-leveling performance.

TABLE IV

ALGORITHM PERFORMANCE FOR DifFERENT TYPES OF FORECASTING ERRORS

\begin{tabular}{lccc}
\hline \hline Forecasting Error & $\begin{array}{c}\text { MSE } \\
(\mathrm{kW})^{2}\end{array}$ & $\begin{array}{c}\text { Variance } \\
(\mathrm{kW})^{2}\end{array}$ & $\begin{array}{c}\text { Average SOC } \\
(\%)\end{array}$ \\
\hline No Error & 5.4 & 139.44 & 99.88 \\
Error in base load & 22.9 & 151.57 & 95.16 \\
Error in number of PEVs & 44.9 & 195.32 & 99.60 \\
Error in mobility parameters & 9.9 & 162.15 & 99.93 \\
Error in all combined & 174.2 & 245.29 & 99.95 \\
\hline \hline
\end{tabular}

3) Transformer Base Loading: Actual loading is not assumed to be exactly the same as the forecast.

In this analysis, the POP value is determined using the assumed models of the mobility parameters and forecasted grid load profile, while the algorithm is run with the actual parameters. Fig. 10(a) illustrates the actual and forecasted loads. The forecasted load refers to the mean of base loading at the first five weekdays of November, and the actual loading refers to the loading at the sixth weekday of November. The result of the proposed algorithm for this case is shown in Fig. 10(b). The algorithm can approximately track the POP value even when there are forecasting errors in the grid base load profile.

The performance summary of the algorithm when abovementioned forecasting errors are considered is presented in Table IV. Here, the same time intervals given in Table II are used to compute MSE and variance. The first row in Table IV refers to the case where all the assumptions are perfect. Among all of the distinct error types, the deviation in number of PEVs returns the worst POP tracking performance. Base load forecasting error yields the most deviation in resulting departure SOC. In the (a)

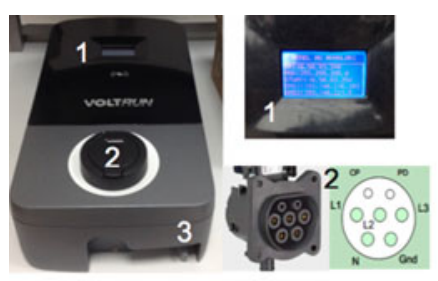

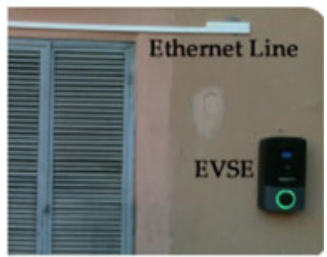

(b)
Fig. 11. Voltrun Mode 2 EVSE. (a) 1: IP settings for network connection, 2: IEC 61851 compatible charging socket, 3: ethernet connection port, (b) EVSE installation with Ethernet Line.

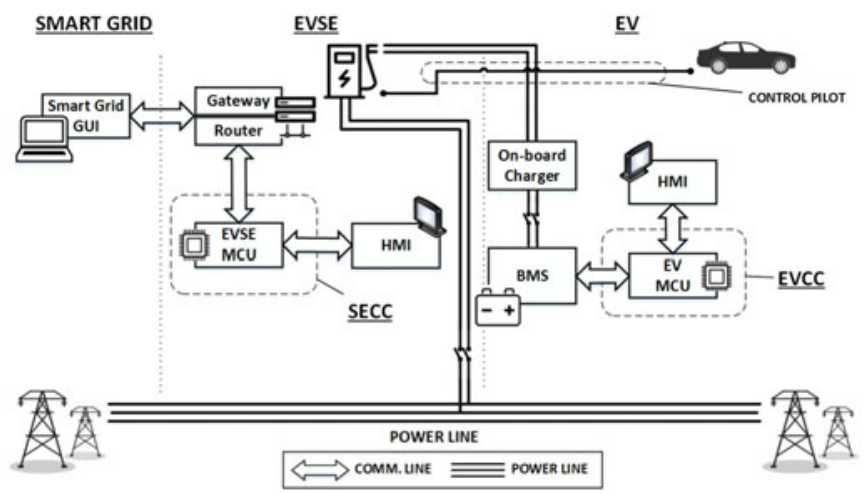

Fig. 12. Implementation diagram of the proposed distributed controller.

meantime, more than $95 \%$ SOC at departure time are acquired under all forecasting error cases. As a result, the proposed charging algorithm also works well when there are forecasting errors within certain limits.

\section{PEV TEst IMPLEMENTATION}

To demonstrate the unidirectional smart charging coordination capability, a BMW i3 EV is tested using a commercial-ofthe-shelf Voltrun EVSE as shown in Fig. 11 [34]. The EVSE has single-phase 7.4-kVA and three-phase 22-kVA power transfer capability, i.e., mode 2 charging according to IEC 61851. BMW i3 is connected to grid via Voltrun EVSE utilizing IEC 61851 compatible Type 2 charging plug. This standard imposes seven connections, with five of them being power lines (L1-L2-L3$\mathrm{N}$-GND) and two of them being $\mathrm{CP}$ and proximity detection pins which are shown in Fig. 11. The unidirectional control is realized using the low-level controlling option utilizing the $\mathrm{CP}$ pin of the charging plug, and by modifying the duty cycle of the $\mathrm{CP}$ as defined in (1). Since BMW i3 has a single-phase on-board charger, it only uses L1-N lines of the charging plug.

The system power and communication architecture is shown in Fig. 12 in detail. Supply equipment communication controller (SECC) in Voltrun EVSE communicates once with the smart grid controller (i.e., a laptop computer) when the vehicle is first plugged-in. At this time, it receives the $P_{\text {valley }}(t)$ information from the grid controller and computes its charging profile according to (6)-(9). Then, it stores this information in EVSE microcontroller unit, and sends back the computed distributed charging profile $P_{c h, i}$ to the utility grid. This 




(a)

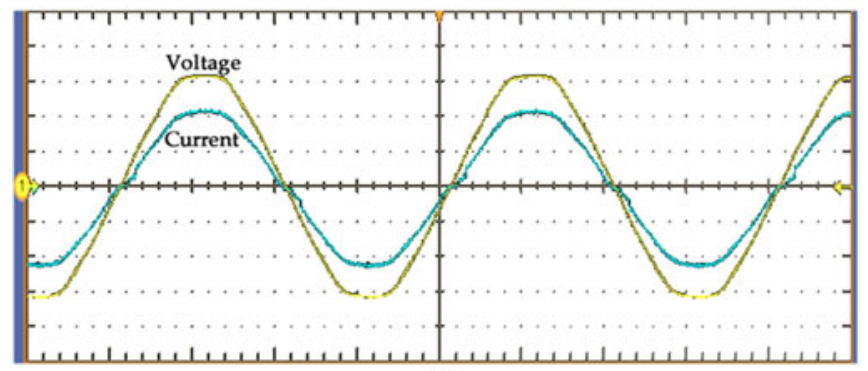

(b)

Fig. 13. Voltage (100 V/div, yellow) and current (20 A/div, cyan) waveforms for BMW i3 on-board charger for (a) $15.8 \mathrm{~A}-3.6 \mathrm{~kW}$, and (b) $31 \mathrm{~A}-$ $7.1 \mathrm{~kW}$. Time: $5 \mathrm{~ms} / \mathrm{div}$.

information stored in SECC constitutes the total charging profile of the vehicle till its departure. Later, SECC communicates the stored charging profile with the electric vehicle communication controller (EVCC) through CP pin as shown in Fig. 12 real time. EVCC controls the on-board charger to track the desired charging profile via in-vehicle CAN bus system.

Fig. 13 shows a snapshot for the implementation of the proposed algorithm using a BMW i3 and Voltrun EVSE. It shows the charging ac voltage and current signals during two different charging cases: $16 \mathrm{~A}$ and $32 \mathrm{~A}$ charging. During the test cases, the single-phase grid voltage is measured to be around $228 \mathrm{~V}$ with a grid frequency of $50 \mathrm{~Hz}$. The related $D$ value for the above operating points are $26.7 \%$ and $53.3 \%$. Fig. 13(a) shows the response of the on-board charger for $16 \mathrm{~A}$ charging command from the EVSE. The EV draws $15.8 \mathrm{~A}$ rms with $3.617-\mathrm{kW}$ charging power consumption. The response time of the EV is less than $1 \mathrm{~s}$ once the comment is sent from the EVSE. Fig. 13(b) shows the response of the on-board charger for 32-A max rms charging command. In this case, the EV draws $31 \mathrm{~A}$ with 7.092-kW charging power. The response time of the EVSE has shown that the algorithm can be implemented on site easily with minimal data transfer requirement.

\section{CONCLUSION}

A distributed smart charging strategy to smooth the load profile has been presented in this paper. PEV user convenience and practicability in real-time applications have also been addressed in the algorithm. Proposed charging strategy has been tested on different loading data in distribution and upstream networks with heuristic charging scenarios and different PEV penetration levels. The effectiveness of the charging strategy has also been demonstrated by comparing with the optimal solution and other state-of-the-art techniques. It is shown that the proposed charging strategy reduces the peak loading from aggregated PEV charging and achieves a significant valley-filling performance independent of the load profile and PEV mobility characteristics.

\section{REFERENCES}

[1] L. P. Fernandez, T. G. S. Roman, R. Cossent, C. M. Domingo, and P. Frias, "Assessment of the impact of plug-in electric vehicles on distribution networks," IEEE Trans. Power Syst., vol. 26, no. 1, pp. 206-213, Feb. 2011.

[2] S. Shafiee, M. Fotuhi-Firuzabad, and M. Rastegar, "Investigating the impacts of plug-in hybrid electric vehicles on power distribution systems," IEEE Trans. Smart Grid, vol. 4, no. 3, pp. 1351-1360, Sep. 2013.

[3] E. Veldman and R. A. Verzijlbergh, "Distribution grid impacts of smart electric vehicle charging from different perspectives," IEEE Trans. Smart Grid, vol. 6, no. 1, pp. 333-342, Jan. 2015.

[4] E. Sortomme, M. M. Hindi, S. D. J. MacPherson, and S. S. Venkata, "Coordinated charging of plug-in hybrid electric vehicles to minimize distribution system losses," IEEE Trans. Smart Grid, vol. 2, no. 1, pp. 198 205, Mar. 2011.

[5] N. Leemput, F. Geth, J. V. Roy, A. Delnooz, J. Buscher, and J. Driesen, "Impact of electric vehicle on-board single-phase charging strategies on a flemish residential grid," IEEE Trans. Smart Grid, vol. 5, no. 4, pp. 18151822, Jul. 2014.

[6] A. S. Masoum, S. Deilami, P. S. Moses, M. A. S. Masoum, and A. AbuSiada, "Smart load management of plug-in electric vehicles in distribution and residential networks with charging stations for peak shaving and loss minimisation considering voltage regulation," IET Gener. Transmiss. Distrib., vol. 5, no. 8, pp. 877-888, Aug. 2011.

[7] Z. Wang and S. Wang, "Grid power peak shaving and valley filling using vehicle-to-grid systems," IEEE Trans. Power Del., vol. 28, no. 3, pp. 18221829, Jul. 2013.

[8] M. C. Kisacikoglu, M. Kesler, and L. M. Tolbert, "Single-phase on-board bidirectional PEV charger for V2G reactive power operation," IEEE Trans. Smart Grid, vol. 6, no. 2, pp. 767-775, Mar. 2015.

[9] SAE Electric Vehicle and Plug-in Hybrid Electric Vehicle Conductive Charge Coupler, SAE International Std. J1772, Jan. 2010.

[10] Electric Vehicle Conductive Charging System- Part-I: General Requirements, International Electrotechnical Commission Std. 61 851-1, 2010.

[11] G. Binetti, A. Davoudi, D. Naso, B. Turchiano, and F. L. Lewis, "Scalable real-time electric vehicles charging with discrete charging rates," IEEE Trans. Smart Grid, vol. 6, no. 5, pp. 2211-2220, Sep. 2015.

[12] Z. Ma, D. S. Callaway, and I. A. Hiskens, "Decentralized charging control of large populations of plug-in electric vehicles," IEEE Trans. Control Syst. Technol., vol. 21, no. 1, pp. 67-78, Jan. 2013.

[13] T. Wu, Q. Yang, Z. Bao, and W. Yan, "Coordinated energy dispatching in microgrid with wind power generation and plug-in electric vehicles," IEEE Trans. Smart Grid, vol. 4, no. 3, pp. 1453-1463, Sep. 2013.

[14] A. Di Giorgio, F. Liberati, and S. Canale, "Electric vehicles charging control in a smart grid: A model predictive control approach," Control Eng. Pract., vol. 22, pp. 147-162, 2014.

[15] C. K. Wen, J. C. Chen, J. H. Teng, and P. Ting, "Decentralized plug-in electric vehicle charging selection algorithm in power systems," IEEE Trans. Smart Grid, vol. 3, no. 4, pp. 1779-1789, Dec. 2012.

[16] A. Malhotra, G. Binetti, A. Davoudi, and I. D. Schizas, "Distributed power profile tracking for heterogeneous charging of electric vehicles," IEEE Trans. Smart Grid, to be published.

[17] Q. Li, T. Cui, R. Negi, F. Franchetti, and M. D. Ilic, "On-line decentralized charging of plug-in electric vehicles in power systems," arXiv: 1106.5063, 2011.

[18] M. C. Kisacikoglu, F. Erden, and N. Erdogan, "A distributed smart PEV charging algorithm based on forecasted mobility energy demand," in Proc. IEEE Global Conf. Signal Inform. Process. Symp. Smart Grid Infrastructure, Washington, DC, USA, Dec. 2016, pp. 1-5.

[19] L. Gan, U. Topcu, and S. H. Low, "Stochastic distributed protocol for electric vehicle charging with discrete charging rate," in Proc. IEEE Power Energy Soc. General Meeting, Jul. 2012, pp. 1-8.

[20] M. C. Kisacikoglu, T. Markel, A. Meintz, J. Zhang, and M. Jun, "EVgrid integration (EVGI) control and system implementation-research overview," presented at the IEEE Appl. Power Electron. Conf. Expo., Long Beach, CA, USA, Mar. 2016. 
[21] G. R. C. Mouli, J. Kaptein, P. Bauer, and M. Zeman, "Implementation of dynamic charging and $\mathrm{V} 2 \mathrm{G}$ using CHAdeMO and CCS/Combo DC charging standard," in Proc. IEEE Transport. Electrific. Conf. Expo., Jun. 2016, pp. 1-6.

[22] S. Y. Derakhshandeh, A. S. Masoum, S. Deilami, M. A. S. Masoum, and M. E. H. Golshan, "Coordination of generation scheduling with PEVs charging in industrial microgrids," IEEE Trans. Power Syst., vol. 28, no. 3, pp. 3451-3461, Aug. 2013.

[23] C. Jin, J. Tang, and P. Ghosh, "Optimizing electric vehicle charging: A customer's perspective," IEEE Trans. Veh. Technol., vol. 62, no. 7, pp. 2919-2927, Sep. 2013.

[24] C. Ahn, C.-T. Li, and H. Peng, "Optimal decentralized charging control algorithm for electrified vehicles connected to smart grid," J. Power Sources, vol. 196, no. 23, pp. $10369-10379,2011$.

[25] L. Gan, U. Topcu, and S. H. Low, "Optimal decentralized protocol for electric vehicle charging," IEEE Trans. Power Syst., vol. 28, no. 2, pp. 940951, May 2013.

[26] F. Erden, M. C. Kisacikoglu, and O. H. Gurec, "Examination of EV-grid integration using real driving and transformer loading data," in Proc. 9th Int. Conf. Elect. Electron. Eng., Nov. 2015, pp. 364-368.

[27] W. Charytoniuk, M. S. Chen, P. Kotas, and P. V. Olinda, "Demand forecasting in power distribution systems using nonparametric probability density estimation," IEEE Trans. Power Syst., vol. 14, no. 4, pp. 1200-1206, Nov. 1999.

[28] M. E. Baran, L. A. A. Freeman, F. Hanson, and V. Ayers, "Load estimation for load monitoring at distribution substations," IEEE Trans. Power Syst., vol. 20, no. 1, pp. 164-170, Feb. 2005.

[29] X. Sun et al., "An efficient approach to short-term load forecasting at the distribution level," IEEE Trans. Power Syst., vol. 31, no. 4, pp. 2526-2537, Jul. 2016.

[30] CVX: Matlab software for disciplined convex programming, Mar. 2017. [Online]. Available: http://cvxr.com/cvx/

[31] Southern California Edison (SCE) website, Apr. 2017. [Online]. Available: https://www.sce.com/005_regul_info/eca/DOMSM13.DLP

[32] Southern California Edison (SCE) website, Apr. 2017. [Online]. Available: https://www.sce.com/005_regul_info/eca/DOMSM14.DLP

[33] U.S. Department of Transportation, 2009 National Household Travel Survey, Apr. 2017. [Online]. Available: http://nhts.ornl.gov/2009/pub/stt.pdf

[34] Zebra electronics, Jul. 2016. [Online]. Available: http://www. zebraelectronics.com

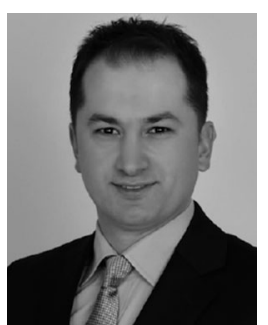

Mithat C. Kisacikoglu (S'04-M'14) received the B.S. degree from Istanbul Technical University, Istanbul, Turkey, in 2005, the M.S. degree from the University of South Alabama, Mobile, AL, USA, in 2007, and the Ph.D. degree from the University of Tennessee, Knoxville, TN, USA, in 2013, all in electrical engineering.

He joined Hacettepe University, Ankara, Turkey, as an Assistant Professor with the Department of Electrical and Electronics Engineering in 2014. He worked at National Renewable Energy Laboratory, Golden, CO, USA, as a Research Engineer between 2015 and 2016. He is currently an Assistant Professor in the Department of Electrical and Computer Engineering, University of Alabama, Tuscaloosa, AL, USA. His research interests include electric vehicles (EVs), EV-grid integration, renewable energy sources, and power electronics converters.

Dr. Kisacikoglu received Postdoctoral Return Fellowship Award from The Scientific and Technological Research Council of Turkey in 2013. He has been an Associate Editor of the IEEE TRANSACTIONS ON INDUSTRY APPLICATIONS since 2014.

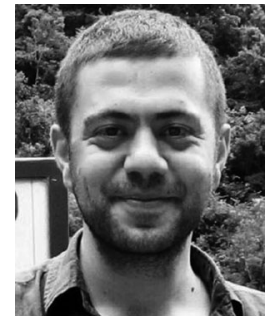

Fatih Erden (M'15) received the B.S. and M.S. degrees from Bilkent University, Ankara, Turkey, in 2007 and 2009, respectively, and the Ph.D. degree from Hacettepe University, Ankara, Turkey, in 2015, all in electrical and electronics engineering.

From 2015 to 2016, he was an Assistant Professor with the Department of Electrical and Electronics Engineering, Atilim University, Ankara, Turkey. He is currently a Visiting Researcher in the Signal Processing Group, Bilkent University. His research interests include signal and image processing, infrared sensors, sensor fusion, multimodal surveillance systems, and EV-grid integration.

Dr. Erden received the Scientific and Technological Research Council of Turkey National M.S. Scholarship Award in 2007, and Bilkent University Full Scholarship in 2003 and 2007.

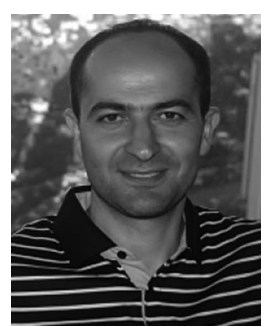

Nuh Erdogan (M'16) received the Ph.D. degree in electrical engineering from the University of Picardie Jules Verne, Amiens, France, in 2005.

From 2007 to 2014, he was a Senior Researcher and R\&D Program Expert with The Scientific and Technological Research Council of Turkey. In 2014, he joined Atilim University, Ankara, Turkey, where he was an Assistant Professor with the Department of Electrical and Electronics Engineering. He is currently a Research Scholar with the Complex Power Electronics Network Laboratory, University of Texas, Arlington, TX, USA. His current research interests include modeling, control, and optimization of electromechanical energy conversion systems, and grid integration of plug-in electric vehicles.

Dr. Erdogan received the Postdoctoral Fellowship Award from TUBITAK in 2015 to conduct research in the U.S. 